\title{
IMAGE SEGMENTATION USING THE DOUBLE MARKOV RANDOM FIELD, WITH APPLICATION TO LAND USE ESTIMATION
}

\author{
Simon P. Wilson, Georgios Stefanou \\ Department of Statistics \\ Trinity College \\ Dublin 2, Ireland
}

\begin{abstract}
We describe the double Markov random field, a natural hierarchical model for a Bayesian approach to model-based textured image segmentation. The model is difficult to implement, even using Markov chain Monte Carlo (MCMC) methods, so we describe an approximation that is computationally feasible. This is applied to a satellite image. We emphasise the valuable additional information about uncertainties in the segmentation that can be gained from the use of MCMC.
\end{abstract}

\section{INTRODUCTION}

The Markov random field has been used in many model based solutions to image segmentation. Here we consider supervised and semi-unsupervised segmentation, where the number of classes in the image is known, but information about their properties is either known or unknown respectively. In a Bayesian approach, we infer the posterior distribution of possible segmentations and, where necessary, any unknown model parameters. This approach is implemented through Markov chain Monte Carlo (MCMC), usually the Gibb's sampler. Although computationally more expensive than many other approaches, MCMC has the advantage that the analysis also yields, through the posterior distribution, information on uncertainty in the segmentation and properties of the texture classes.

In this paper we describe one such model, the double. Markov random field, and how it may be used in a Bayesian solution to the segmentation problem. We look at the sort of information this procedure yields that may be of use in the analysis of satellite images.

\section{THE MODEL}

Let $S$ be a rectangular lattice of pixel sites, and define an image to be an array of grey values $\left(x_{s}\right)_{s \in \mathcal{S}}$ and labels $\left(y_{s}\right)_{s \in \mathcal{S}}$,

Research made possible through Project MOUMIR, a 5th Framework funded Research Network. identifying the class of each pixel. Assume $R$ classes in the image, each a Markov random field $T^{r}$ defined on all of $\mathcal{S}$, parameterised by $\theta_{r}$, with neighbourhood system $\mathcal{C}_{r}$. This allows texture based segmentation. The label process is another Markov random field $Y$, parameterised by $\beta$ and with neighbourhood system $\mathcal{C}_{Y}$. All the fields are independent conditional on model parameters and their probability distributions have the Gibb's representations $P\left(T^{r}=t \mid \theta_{r}\right)=$ $\exp \left[-\sum_{c \in \mathcal{C}_{r}} V_{r, c}\left(t ; \theta_{r}\right)\right] / Z_{r}\left(\theta_{r}\right)$, and $P(Y=y \mid \beta)=$ $\exp \left[-\sum_{c \in \mathcal{C}_{Y}} V_{Y, c}(y ; \beta)\right] / Z_{Y}(\beta)$. The observed image is the collage $X=\left(T_{s}^{Y s}\right)_{s \in \mathcal{S}}$, and the joint distribution for $X$ and $Y$ is:

$$
\begin{aligned}
P(X=x, Y & \left.=y \mid \theta_{1}, \ldots, \theta_{R}, \beta\right) \\
& =P(Y=y \mid \beta) \prod_{r=1}^{R} P\left(T_{S_{r}}^{r}=x_{S_{r}} \mid \theta_{r}\right),
\end{aligned}
$$

where $S_{r}=\left\{s \in \mathcal{S} \mid y_{s}=r\right\}$ and $T_{S_{r}}^{r}, x_{S_{r}}$ denote $T^{r}$ and $x$ restricted to $S_{r}$.

Evaluation of $P\left(T_{S_{r}}^{r}=x_{S_{r}} \mid \theta_{r}\right)$ is usually intractable because it requires marginalisation of $P\left(T^{r} \mid \theta_{r}\right)$ over $\mathcal{S}-$ $\mathcal{S}_{r}$. This is solved by assuming a free boundary for pixels at the edge of $\mathcal{S}_{r}$, giving

$$
P\left(T_{S_{r}}^{r}=x_{S_{r}} \mid \theta_{r}\right)=\frac{\exp \left[-\sum_{c \in \mathcal{C}_{r} \mid c \subset S_{r}} V_{r, c}\left(x_{c} \mid \theta_{r}\right)\right]}{Z_{S_{r}}\left(\theta_{r}\right)} .
$$

Equations 1 and 2 define the double Markov random field. Reference to this model was first made in [1].

\section{THE SEGMENTATION ALGORITHM}

Segmentation is based on the posterior density

$$
\begin{aligned}
& P\left(y, \theta_{1}, \ldots, \theta_{R}, \beta \mid x\right) \\
& \propto P\left(X=x, Y=y \mid \theta_{1}, \ldots, \theta_{R}, \beta\right) \pi\left(\theta_{1}, \ldots, \theta_{R}, \beta\right),
\end{aligned}
$$

in the semi-supervised case, for a prior distribution $\pi$, or $P\left(y \mid \theta_{1}, \ldots, \theta_{R}, \beta, x\right)$ in the supervised case. A "best" sin- 
gle segmentation $y^{*}$ is then selected from the posterior distribution. Two are predominant: the maximum a posteriori (MAP), $y^{*}=\arg \max _{y} P(Y=y \mid x)$, or the marginal posterior mode (MPM), $y_{s}^{*}=\arg \max _{y_{s}} P\left(Y_{s}=y_{s} \mid x\right)$. The former is found by stochastic maximisation, usually simulated annealing in tandem with Gibb's sampling, and the latter by Gibb's sampling of the posterior, and picking the most frequently generated label at each site after convergence is deemed to have occurred (see [2] or more specifically' [3]).

Equations 1 and 2 are the ideal model but cannot be readily used because the partition functions $Z_{S_{r}}\left(\theta_{r}\right)$ cannot be computed. Further, Gibb's sampling directly from Equation 3 is not possible because the local interaction structure between labels is lost a posteriori. We modify Equation 2 to define posterior full conditional distributions on $Y$ that admit a Gibb's formulation, using the idea of pseudolikelihood [4]. In [5], four other possible modifications to the ideal model are described and compared; the one we describe here was judged the best performing.

The segmentation algorithm is then the Gibb's sampler: a sample from the full conditional distribution of each label is taken, and from the full conditional of each model parameter if necessary. This is repeated until convergence to the posterior distribution is thought to have been reached in the MPM case, or is used in tandem with simulated annealing in the MAP case.

\section{PSEUDO-LIKELIHOOD APPROXIMATION}

One approach is to consider the grey levels to be composed of a set of overlapping windows $\left(W_{s}\right)_{s \in \mathcal{S}}$ of size $n \times n$. Within each window the texture $Y_{s}$ is assumed. The minimum size of window that can be considered without losing textural information is one that contains the neighbourhood of the central pixel. Thus the likelihood for grey levels in class $r$ is assumed to be:

$$
\begin{aligned}
P\left(T_{S_{r}}^{r}=\right. & \left.x_{S_{r}} \mid \theta_{r}\right) \\
= & \prod_{s \in S_{r}} \frac{\exp \left[-\sum_{c \in \mathcal{C}_{r} \mid i \in c} V_{r, c}\left(x_{c} \mid \theta_{r}\right)\right]}{C_{s}(x)},
\end{aligned}
$$

where

$$
C_{s}(x)=\int \exp \left(-\sum_{c \in \mathcal{C} \mid i \in c} V_{r, c}\left(x_{c} \mid \theta_{r}\right)\right) d x_{i} .
$$

This is equivalent to using the pseudo-likelihood approximation to the true likelihood. Under Gaussian CAR texture models and a Potts model for the labels, the full conditionals of the posterior of $Y$ are:

$$
\begin{gathered}
P\left(y_{s}=r \mid x, y_{j}, j \neq s, \theta_{r}, \beta\right) \propto \frac{1}{\sqrt{2 \pi \sigma_{r}^{2}}} \\
\times \exp \left[-\frac{\left\{x_{s}-\mu_{r}-\sum_{k=1}^{K} \sum_{j|<s, j\rangle_{k}} \phi_{r_{k}}\left(x_{j}-\dot{\mu}_{r}\right)\right\}^{2}}{2 \sigma_{r}^{2}}\right] \\
\times \exp \left[\beta \sum_{k=1}^{2} \sum_{j \mid<s, j>_{k}} \delta\left(r-y_{j}\right)\right],
\end{gathered}
$$

which can be easily simulated from.

In the semi-unsupervised case, we must also sample from the full conditional distributions of the model parameters. This we also do via a pseudo-likelihood approximation. With uniform prior distributions over some suitable range on all texture parameters, the full conditionals for $\theta_{r}$ are then proportional to

$$
P L\left(\theta_{r} ; x, y, \beta\right)=\prod_{s \in \mathcal{S}_{r}} P\left(y_{s}=r \mid x, y_{j}, j \neq s, \theta_{r}, \beta\right) .
$$

In the case of Gaussian CAR parameters, the range of allowable values is determined in [6] and the full conditionals are given in [3, Appendix 2].

\section{APPLICATION TO LAND USE ESTIMATION}

The image at the left of Figure 1 is of an agricultural region of Holland at a resolution of $10 \mathrm{~m}$ per pixel. The semiunsupervised MPM segmentation algorithm was applied. It algorithm was run for 1000 iterations and the results are based on the last 600 iterations. Figure 2 displays the results for an MPM segmentation into 4 classes, as well as some of the additional information available from the MCMC procedure. Figure 3 is the entropy in marginal posterior distribution of each label, that is

$$
e_{s}=-\sum_{\substack{j=1 \\ \hat{p}_{0 j}>0}}^{R} \hat{p}_{s j} \log \left(\hat{p}_{s j}\right)
$$

where $\hat{p}_{s j}$ is the proportion of times $y_{s}$ was sampled as class $j$, with lighter colours indicating higher entropy. This gives a measure of uncertainty in the class of each pixel, and we see that class 1 (coloured black in the segmentation) has the lowest uncertainty in general, and the highest uncertainty occurs at the borders between regions.

By looking at the relative proportion of values sampled, estimates of posterior distributions of parameters can be made. The top of Figure 4 is an estimate of the posterior distribution of the mean intensity of class 1 . Similar plots can be made for all other model parameters, although we note that 
the pseudo-likelihood may not be a good approximation for the posterior of correlation parameters of the CAR model. Having recorded at each iteration the number of pixels in each class, we can construct the lower plot of Figure 4, being an estimate of the posterior distribution of the percentage of pixels that are in this class, something that has clear use in land use estimation. Equally easy to calculate from the MCMC output is the full posterior distribution of label class for each pixel.

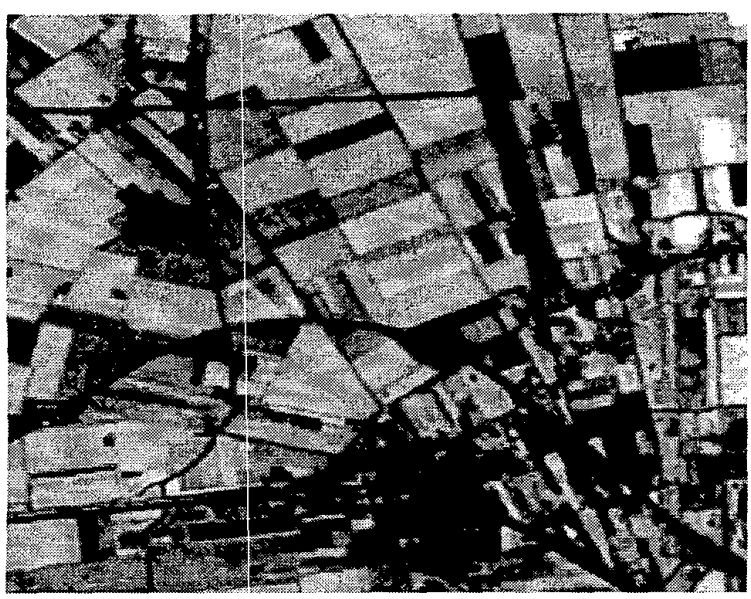

Fig. 1. Radar image of an agricultural region of Holland.

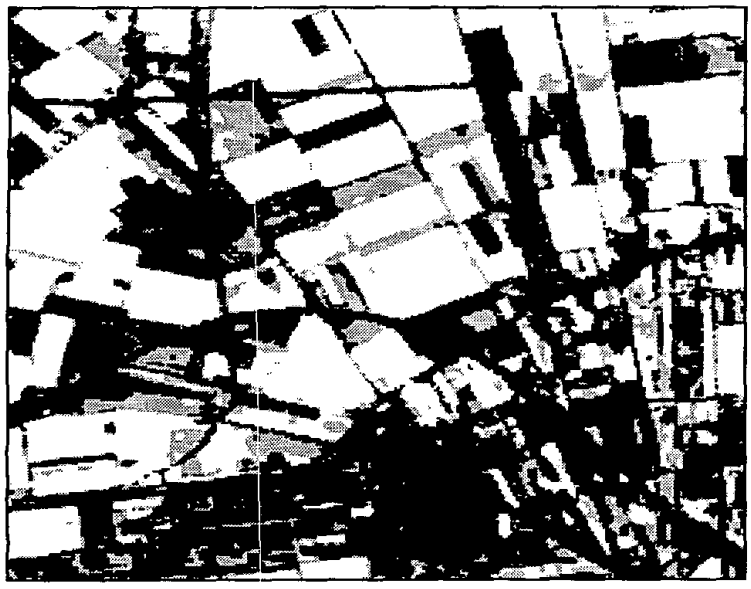

Fig. 2. MPM segmentation of the image in Figure 1 into 4 classes.

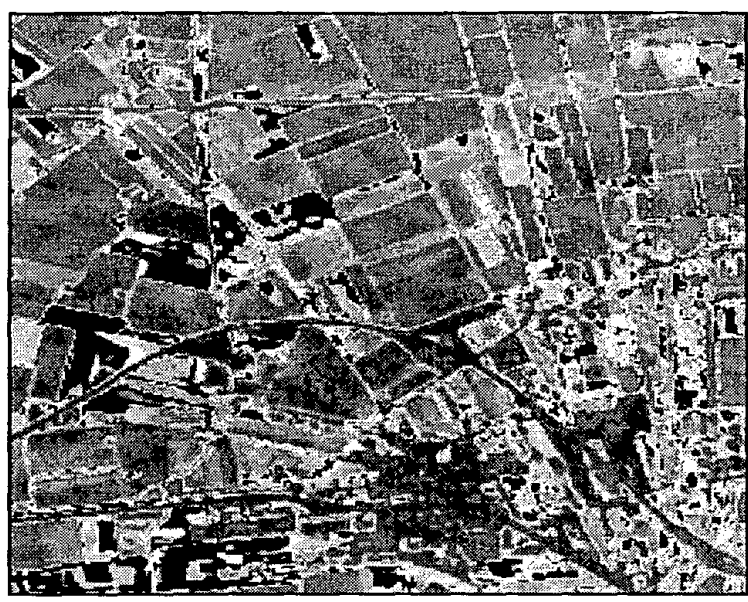

Fig. 3. Entropy of MPM segmentation of the image in Figure 1 into 4 classes.

\section{CONCLUDING REMARKS}

The double Markov random field model is, we contend, a natural way to combine Markov random field models for textures and labels. We have described how this model may be modified in order to readily implement a segmentation algorithm through Gibb's sampling. We emphasise that the power of the MCMC approach is not in its speed but in the additional information on levels of uncertainty in the segmentation that can be calculated, as shown in Figures 3 and 4.

\section{ACKNOWLEDGEMENTS}

The satellite image of Holland is courtesy of the Centre $\mathrm{Na}$ tional d'Etudes Spatiales (the French Space Agency).

\section{REFERENCES}

[1] J. Zhang, J. W. Modestino, and D. A. Langan, "Maximum likelihood parameter estimation for unsupervised stochastic model-based image segmentation," IEEE Trans. Image Processing, vol. 3, pp. 404-419, 1994.

[2] S. Geman and D. Geman, "Stochastic relaxation, Gibb's distributions and the Bayesian restoration of images," IEEE Trans. Pattern Anal. Machine Intell., vol. PAMI-6, pp. 721-741, 1984.

[3] D. E. Melas, A Bayesian Approach to the Segmentation of Textured Images, Ph.D. thesis, Statistics Department, University of Dublin, 1999. 
[4] J. Besag, "Spatial interaction and the statistical analysis of lattice systems (with discussion)," J. Roy. Statist. Soc. B, vol. 36, pp. 192-236, 1974.

[5] D. E. Melas and S. P. Wilson, "Double Markov random fields and Bayesian image segmentation," Tech. Rep. 00/03, Department of Statistics, Trinity College Dublin, 2000.

[6] S. Lakshmanan and H. Derin, "Valid parameter space for 2-d Gaussian Markov random fields," IEEE Trans. Inform. Theory, vol. 39, pp. 703-709, 1993.
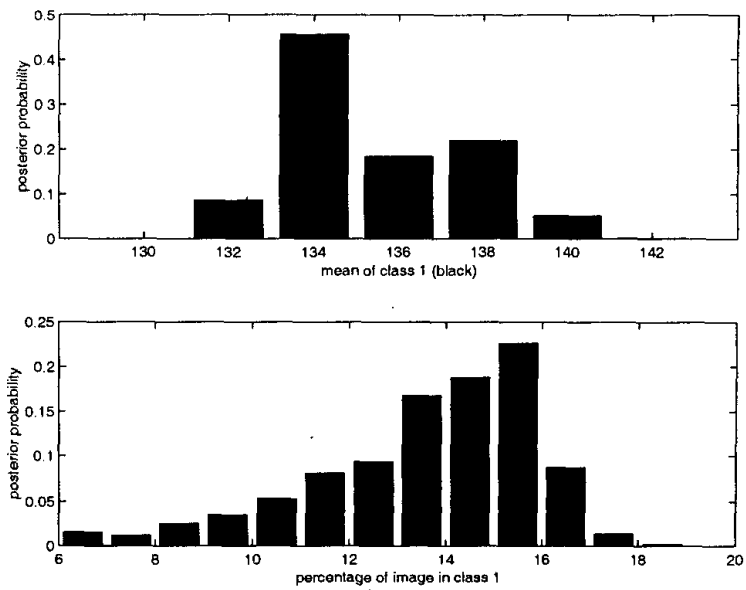

Fig. 4. Estimates of the posterior density of: top, the mean intensity for class 1 (coloured black in Figure 2) and bottom, the percentage of pixels in class 1 . 